\title{
Incidence of register-based diabetes 10 years after a stepwise diabetes screening programme: the ADDITION-Denmark study
}

\author{
Signe S. Rasmussen ${ }^{1}$ - Nanna B. Johansen ${ }^{2,3} \cdot$ Daniel R. Witte ${ }^{3,4} \cdot$ Knut Borch-Johnsen $^{5}$. \\ Annelli Sandbaek $^{4}$ - Torsten Lauritzen ${ }^{4}$ - Marit E. Jørgensen ${ }^{2,6}$
}

Received: 11 September 2015 / Accepted: 22 January 2016 / Published online: 8 February 2016

(C) Springer-Verlag Berlin Heidelberg 2016

\begin{abstract}
Aims/hypothesis Screening programmes for type 2 diabetes inevitably find more people at high risk of developing diabetes than people with undiagnosed prevalent diabetes. We describe the incidence of diabetes for risk groups according to advancement in a screening process.

Methods In 2001-2006, a diabetes screening programme based on the Danish diabetes risk score and measures of $\mathrm{HbA}_{1 \mathrm{c}}$ and glucose was carried out in Danish general practices. The present study includes 13,249 individuals with low diabetes risk scores and 22,726 with high diabetes risk scores but no diabetes according to WHO 1999 criteria. Seven incremental levels of diabetes risk were defined and followed for incident diabetes recorded in the Danish National Diabetes
\end{abstract}

Signe S. Rasmussen and Nanna B. Johansen contributed equally to this study.

Electronic supplementary material The online version of this article (doi:10.1007/s00125-016-3887-6) contains peer-reviewed but unedited supplementary material, which is available to authorised users.

Signe S. Rasmussen

signe.sabine.saetre.rasmussen.01@regionh.dk

1 Department of Cardiology, Nephrology and Endocrinology H, Nordsjællands University Hospital, Dyrehavevej 29,

3400 Hillerød, Denmark

2 Steno Diabetes Center, Gentofte, Denmark

3 The Danish Diabetes Academy, Odense, Denmark

4 Department of Public Health, Section for General Practice, Aarhus University, Aarhus, Denmark

5 Holbæk Hospital, Holbæk, Denmark

6 Center for Health Research in Greenland, Southern Denmark University, Odense, Denmark
Register until December 2012. For each group, cumulative diabetes incidence was calculated. Incidence rates and rate ratios were estimated by Poisson regression analyses.

Results After 10 years of follow-up 1,164 new diabetes cases were registered. Incidence rates were 1.0, 4.2, 14.5, 28.8 and 52.6 per 1,000 person-years in individuals at low risk and in those with normal glucose tolerance, impaired fasting glucose, impaired glucose tolerance and one diabetic glucose value, respectively. For each step in the screening algorithm, the risk of developing diabetes was higher than in the previous step. Conclusions/interpretation The risk of developing clinical diabetes in people who screen negative for diabetes depends on the level of risk stratification at screening, even at lower risk levels. This risk increases markedly in the presence of impaired glucose regulation. These results can inform policy recommendations concerning prevention strategies following screening.

Keywords $\mathrm{HbA}_{1 \mathrm{c}} \cdot$ High risk $\cdot$ Impaired fasting glucose $\cdot$ Impaired glucose tolerance $\cdot$ Incidence $\cdot$ Normal glucose tolerance $\cdot$ Register $\cdot$ Screening $\cdot$ Type 2 diabetes mellitus

$\begin{array}{ll}\text { Abbreviations } \\ \text { 2hBG } & \begin{array}{l}\text { 2 h blood glucose } \\ \text { ADglo-Danish-Dutch Study of Intensive } \\ \text { ADDITION }\end{array} \\ & \begin{array}{l}\text { Treatment in People with Screen-Detected } \\ \text { Diabetes in Primary Care }\end{array} \\ \text { FBG } & \text { Fasting blood glucose } \\ \text { IFG } & \text { Impaired fasting glucose } \\ \text { IGT } & \text { Impaired glucose tolerance } \\ \text { IQR } & \text { Interquartile range } \\ \text { NGT } & \text { Normal glucose tolerance } \\ \text { RBG } & \text { Random blood glucose }\end{array}$




\section{Introduction}

Screening programmes for type 2 diabetes inevitably find more people at high risk of developing diabetes than people with undiagnosed prevalent diabetes. The different screening strategies for type 2 diabetes identify different dysglycaemic subgroups $-\mathrm{HbA}_{1 \mathrm{c}} 6.0-6.4 \%$ (42-47 mmol$\left./ \mathrm{mol}\right)$, impaired glucose tolerance (IGT) or impaired fasting glucose (IFG)as well as groups with normoglycaemia at high diabetes risk based on the presence of multiple risk factors as assessed in risk scores. Clinical management and re-screening strategies for this heterogeneous group with intermediate or high diabetes risk vary widely, due to a limited evidence base.

There is strong evidence that progression to diabetes is preventable with intensive lifestyle modification or pharmacological interventions in individuals with IGT [1-5]. It remains unclear whether a similar effect is also achievable in those with IFG or intermediate $\mathrm{HbA}_{1 \mathrm{c}}$.

Regarding healthcare planning, there is a surprising scarcity of data on diabetes incidence in the general population. A marked difference is seen between incidence rates based on self-reported or register-based diagnosed diabetes (ranging from around 0.5 to 10 per 1,000 person-years depending on age) [6-8] and incidence rates based on sequentially repeated diagnostic testing (ranging from around 7 to 20 per 1,000 person-years) [9-13]. A meta-analysis found that the progression rate to diabetes was $40-75$ per 1,000 person-years in individuals with IFG or IGT [14], giving annualised relative risks of diabetes development for the different dysglycaemic groups of between 5 and 12 [15]. When individuals with IFG and IGT are identified by a highrisk screening programme, though, it appears that the risk of incident diabetes is higher than that found in general population-based studies [16]. This indicates that the evidence on diabetes incidence from classic population-based studies may provide unrealistic estimates for healthcare policy making such as rescreening strategies.

In most screening programmes, the diagnostic test (fasting blood glucose [FBG], OGTT or $\mathrm{HbA}_{1 \mathrm{c}}$ ) is preceded by a questionnaire-based risk assessment which serves to focus the diagnostic test on individuals most at risk. This process of high-risk screening or case-finding/opportunistic screening for diabetes, however, also identifies people who have high risk scores but who do not have impaired glucose regulation. Clinically relevant evidence on the differentiated risk of developing diabetes in such everyday screening situations is lacking and might be essential to guide physicians on how to advise their patients.

We aimed to examine the difference in incidence of clinical diabetes as registered in the Danish National Diabetes Register between groups at several incremental levels of increasing diabetes risk, identified during the screening programme of the Anglo-Danish-Dutch Study of Intensive Treatment in People with Screen-Detected Diabetes in Primary Care (ADDITION)-Denmark study.

\section{Methods}

Study population The ADDITION-Europe study is a population-based study with the overall aim to evaluate whether screening and subsequent optimised intensive treatment of diabetes and associated risk factors improve the prognosis for individuals with undiagnosed type 2 diabetes [17]. The study was initiated in Denmark and also included centres in the UK and the Netherlands. In Denmark the study was conducted in general practices in five counties. General practitioners participating in the study were randomised to provide standard care or intensive pharmacological and nonpharmacological treatment of people with screen-detected diabetes. Screening for diabetes took place in 2001-2006 in 190 general practices in which all those aged 40-69 years and without known diabetes received a diabetes risk score questionnaire (the Danish diabetes risk score) [18].

A pilot study was conducted in five general practices prior to initiation of the ADDITION-Denmark study. One aim of this pilot study was to enable cut-off levels (concerning $\mathrm{HbA}_{1 \mathrm{c}}$, random blood glucose [RBG] and FBG) for advancing in the stepwise screening programme. In the ADDITIONDenmark study, the screening procedure consisted of four steps (Fig. 1): based on the Danish diabetes risk score, 28,035 people were identified as having a high risk of diabetes and underwent measurements of RBG and $\mathrm{HbA}_{1 \mathrm{c}}$. If $\mathrm{RBG}$ was $<5.5 \mathrm{mmol} / 1$ and $\mathrm{HbA}_{1 \mathrm{c}}$ was $<5.8 \%(40 \mathrm{mmol} / \mathrm{mol})$, no further glucose measurements were performed. If RBG was $\geq 5.5 \mathrm{mmol} / 1$ and $\mathrm{HbA}_{1 \mathrm{c}}$ was $<5.8 \%(40 \mathrm{mmol} / \mathrm{mol})$, FBG was measured. If this $\mathrm{FBG}$ was $<5.6 \mathrm{mmol} / \mathrm{l}$, no further tests were carried out; if, however, the FBG measurement was between 5.6 and $6.1 \mathrm{mmol} / \mathrm{l}, 2 \mathrm{~h}$ blood glucose $(2 \mathrm{hBG})$ was measured after a $75 \mathrm{~g}$ OGTT. If $\mathrm{HbA}_{1 \mathrm{c}}$ was $\geq 5.8 \%(40 \mathrm{mmol} /$ mol), FBG and $2 \mathrm{hBG}$ were measured on a second day. Diabetes was defined by the 1999 WHO criteria using capillary blood glucose [19] when RBG was $\geq 11.1 \mathrm{mmol} / \mathrm{l}$, FBG was $\geq 6.1 \mathrm{mmol} / 1$ or $2 \mathrm{hBG}$ was $\geq 11.1 \mathrm{mmol} / 1$. To be included in the interventional part of the ADDITIONEurope study this initial diabetic value had to be confirmed by an OGTT, on a separate day, as 'clinical diabetes'. In order to establish a group at low risk of diabetes, a subgroup $(n=32$, 894) was asked to return their risk score questionnaire regardless of their score. This identified 13,249 people with a low diabetes risk $(<5$ points on the risk score) [20].

On the basis of how far they advanced in the programme, those who completed the screening programme and were not diagnosed with screen-detected clinical diabetes were classified into seven groups of increasing diabetes risk (Fig. 1). 


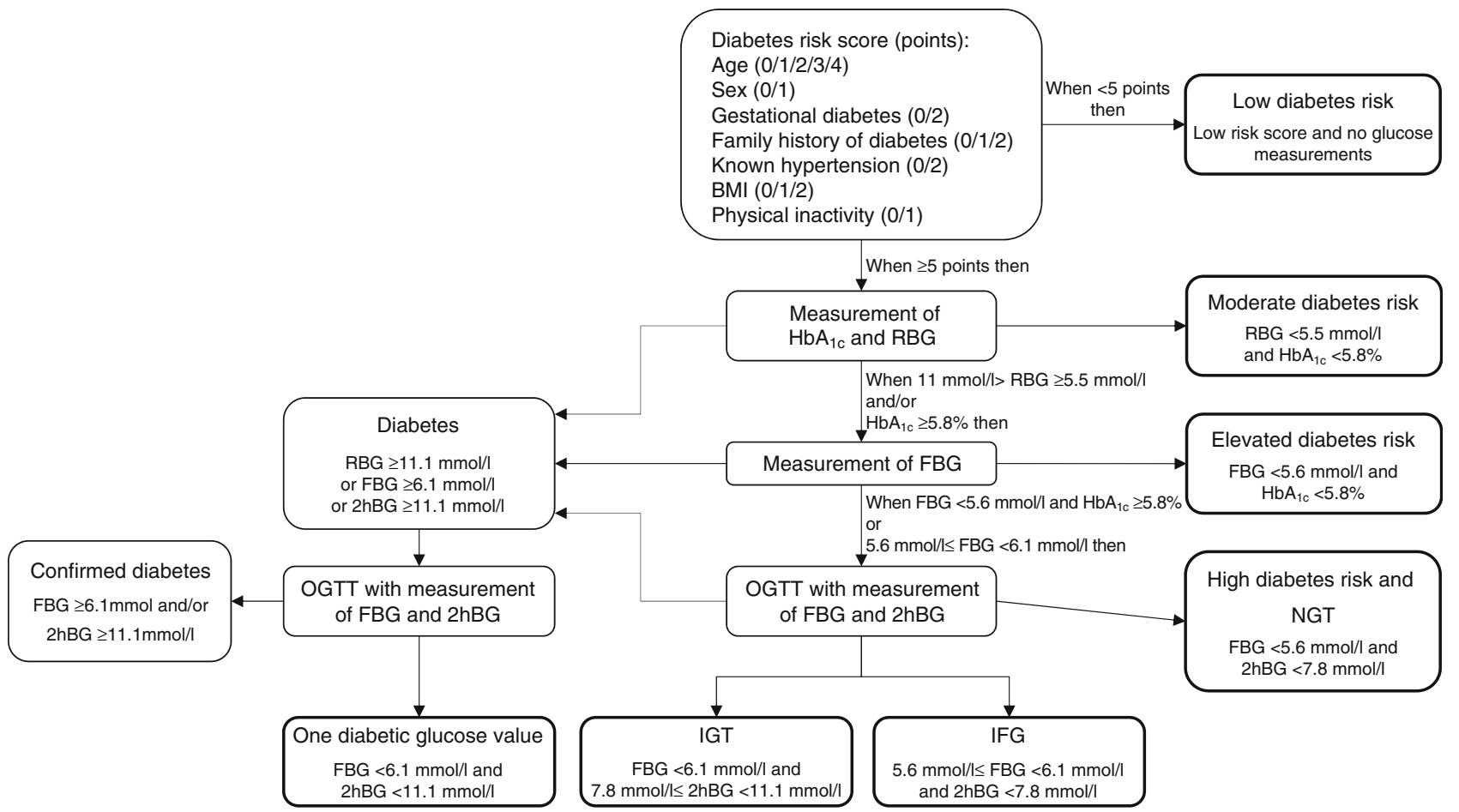

Fig. 1 Stepwise screening procedure of ADDITION-Denmark study. To convert values for per cent $\mathrm{HbA}_{1 \mathrm{c}}$ into mmol/mol, subtract 2.15 and multiply by 10.929

- The first group had a low diabetes risk (risk score $<5$ ); no blood glucose measurements were therefore carried out $(n=13,249)$.

The following risk groups had a risk score $\geq 5$ :

- Moderate diabetes risk was defined as $\mathrm{RBG}<5.5 \mathrm{mmol} / \mathrm{l}$ and $\mathrm{HbA}_{1 \mathrm{c}}<5.8 \%$ (40 mmol/mol); no further glucose measurements were done $(n=13,037)$.

- Elevated diabetes risk was defined as $5.5 \leq \mathrm{RBG}$ $<11.1 \mathrm{mmol} / \mathrm{l}, \mathrm{HbA}_{1 \mathrm{c}}<5.8 \%(40 \mathrm{mmol} / \mathrm{mol})$ and $\mathrm{FBG}$ $<5.6 \mathrm{mmol} / \mathrm{l} ; 2 \mathrm{hBG}$ was not measured $(n=4,220)$.

In the following high-risk groups an OGTT with measurement of FBG and $2 \mathrm{hBG}$ defined the glucose tolerance status.

- High diabetes risk with normal glucose tolerance (NGT) was defined as $\mathrm{FBG}<5.6 \mathrm{mmol} / \mathrm{l}$ and $2 \mathrm{hBG}<7.8 \mathrm{mmol} / 1$ $(n=2,628)$.

- IFG was defined as $5.6 \mathrm{mmol} / \mathrm{l} \leq \mathrm{FBG}<6.1 \mathrm{mmol} / \mathrm{l}$ and $2 \mathrm{hBG}<7.8 \mathrm{mmol} / \mathrm{l}(n=913)$.

- IGT was defined as FBG $<6.1 \mathrm{mmol} / \mathrm{l}$ and $7.8 \mathrm{mmol} / \mathrm{l} \leq$ $2 \mathrm{hBG}<11.1 \mathrm{mmol} / 1 \quad(n=1,306)$. (Isolated IGT was defined as $\mathrm{FBG}<5.6 \mathrm{mmol} / \mathrm{l}$ and $7.8 \mathrm{mmol} / \mathrm{l} \leq 2 \mathrm{hBG}$ $<11.1 \mathrm{mmol} / 1, n=765$; combined IFG and IGT was defined as $5.6 \mathrm{mmol} / \mathrm{l} \leq \mathrm{FBG}<6.1 \mathrm{mmol} / \mathrm{l}$ and $7.8 \mathrm{mmol} / \mathrm{l} \leq$ $2 \mathrm{hBG}<11.1 \mathrm{mmol} / \mathrm{l}, n=541$ ).
- The group with one diabetic blood glucose measurement that was not confirmed on the second test had RBG $\geq 11.1 \mathrm{mmol} / 1$ or one measurement of $\mathrm{FBG} \geq 6.1 \mathrm{mmol} / 1$ or $2 \mathrm{hBG} \geq 11.1 \mathrm{mmol} / \mathrm{l}(n=622)$.

In total, 35,975 people were classified into seven groups.

Danish National Diabetes Register The Danish National Diabetes Register [21] is a validated database of people with diabetes of either type. It is based on the Danish Civil Registration System, in which each inhabitant has a Central Person Register number (a unique identification system of Danish citizens including survival status), the National Patient Register, the National Health Service Register and the Danish National Prescription Registry, constructed and maintained by the National Board of Health. Data have been included since 1997, and the National Diabetes Register is updated annually.

Date of inclusion in the register is the date when a person first meets one of the following inclusion criteria. (1) National Patient Register: hospital contacts with diabetes diagnosis by ICD-10 (www.who.int/classifications/icd/en/) codes DE10, DE11, DE12, DE13, DE14, DO24 and DH360. There is no national coding available for contacts in general practice. (2) National Health Service Register: registering services but not values of the services, i.e. blood glucose values; five blood glucose measurements in 1 year; two blood glucose 
measurements per year in 5 consecutive years; registration of chiropody (service for a person with diabetes). (3) Danish National Prescription Registry: redeemed prescription within 6 months at least twice for insulin, or at least twice for glucagon-like peptide 1 analogue or oral glucose-lowering medication. Inclusion date is the date for the second redeemed prescription.

As individuals participating in our screening programme underwent several glucose measurements due to the screening algorithm, we excluded the two criteria based on blood glucose measurements in our data analysis in order to avoid cases representing false-positive inclusion of those with frequent measurements of blood glucose without having diabetes [22]. This gives a conservative (specific but not very sensitive) definition of diabetes.

Data analyses Individuals who participated in the screening programme were grouped into the seven risk groups shown in Fig. 1. Data on these individuals were linked to the Danish National Diabetes Register updated to December 2012.

Baseline description data were summarised as medians (interquartile range [IQR]) or percentages. The cumulative risk of diabetes since the date of screening to the end of December 2012 was calculated for the seven groups. People were followed from study entry (screening) until the date of diabetes diagnosis, death or the last update of the diabetes register (31 December 2012), whichever occurred first. The corresponding incidence rates of diabetes and rate ratios, with the low-risk group as reference, were calculated using Poisson regression analyses, taking death before end of follow-up into account. A sensitivity analysis including age at screening in the model was performed, as age is a well-known, strong predictor of diabetes. To illustrate diabetes incidence over time, predicted incidence rates were modelled as a function of time since screening for each group. Statistical analyses were performed using SAS software (version 9.3; SAS Institute, Cary, NC, USA) and R (version 3.1.1; R Foundation for Statistical Computing, Vienna, Austria). The ADDITION study has been approved by the local Aarhus county ethics committees (reference number 20000183). The study complied with the Helsinki Declaration and participants gave written informed consent. Access to the Danish National Diabetes Register and the National Patient Register was approved by the Danish Data Protection Agency.

\section{Results}

The 35,975 screened individuals had a median age of 56.2 years (IQR 49.2-62.7) and 46.0\% were men (Table 1). During a median follow-up of 9.9 years (IQR 7.8-10.8), 1,164 people were registered with diabetes and 2,208 were censored due to death before diabetes diagnosis. Those censored through death were distributed evenly across groups. Censoring due to emigration is not considered relevant as the number of emigrants is very low.

The cumulative diabetes incidence was $<1 \%$ in the group with low diabetes risk, based on the risk score questionnaire; $1-4 \%$ in intermediate diabetes risk groups, including high-risk NGT; and $12 \%, 22 \%$ and $34 \%$ in individuals with IFG, IGT and one diabetic glucose value, respectively (Table 2). The overall diabetes incidence rate was 3.51 (95\% CI 3.32, 3.72) per 1,000 person-years. The low-risk group had a diabetes incidence rate of 0.99 per 1,000 person-years. Diabetes incidence rates were two to four times higher in individuals with intermediate diabetes risk compared with those with low diabetes risk (Table 2), whereas the rate ratios among those with impaired glucose regulation were markedly higher: 14.6, 29.1 and 53.1 for IFG, IGT and one diabetic glucose value, respectively (Table 2). Adjusting for age did not change the rate ratios markedly (electronic supplementary material [ESM] Table 1).

Figure 2 shows the predicted diabetes incidence rates as a function of time over 10 years, after screening for diabetes, for the seven risk groups separately. After 1 year, the riskstratified groups tended to cluster into four patterns (Fig. 2). For those with one diabetic glucose value, the incidence was higher than for the other risk groups even though there was an exponential decrease during the follow-up period. For those with IFG or IGT, the incidence increased in the first 2 years after screening and subsequently decreased, whereas the incidence peaked after 5-6 years in the intermediate-risk groups. By contrast, diabetes incidence was more or less constant during the first 6 years in the lowest risk group. After 8 years of follow-up, there were only 19 diabetes cases in total, explaining the shapes of the curves and wide CIs at the end of the follow-up period.

\section{Discussion}

On the basis of linkage of the baseline risk classification from a stepwise screening study - the ADDITION-Denmark Study — with the Danish National Diabetes Register, we found that each incremental baseline risk level corresponded to a higher risk of register-based diabetes during a median of 10 years of follow-up. The incidence rate of register-based diabetes ranged from $1.0(95 \%$ CI $0.8,1.2)$ per 1,000 person-years in the low-risk group to 52.6 (95\% CI 46.0, 60.1) among those with a single diabetic value at screening, not confirmed during a second OGTT. Hence, compared with the low-risk group, the risk of developing diabetes was more than fifty times higher in the group with one diabetic glucose value.

Targeted screening and case-finding programmes focused on high-risk individuals are increasingly common worldwide. 


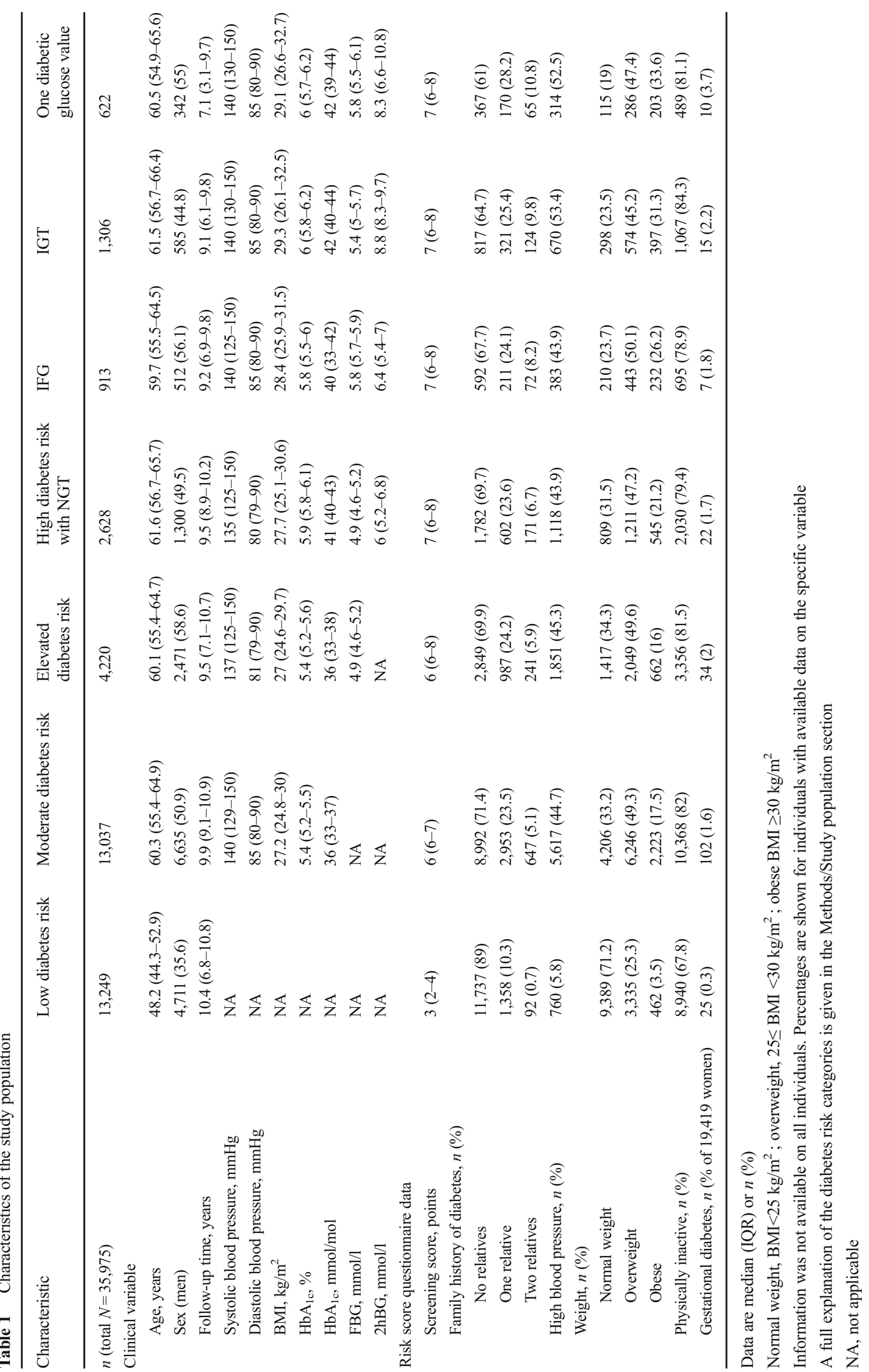


Table 210 year diabetes incidence and rate ratios

\begin{tabular}{llllll}
\hline Risk stratification & $n$ & $\begin{array}{l}\text { Cumulative diabetes } \\
\text { incidence, } n(\%)\end{array}$ & Person-years & $\begin{array}{l}\text { Incidence per 1,000 person-years } \\
(95 \% \text { CI })\end{array}$ & $\begin{array}{l}\text { Rate ratio with low risk as } \\
\text { reference group (95\% CI) }\end{array}$ \\
\hline Low diabetes risk & 13,249 & $123(0.93)$ & 124,169 & $0.99(0.83,1.18)$ & Reference \\
Moderate diabetes risk & 13,037 & $237(1.82)$ & 124,621 & $1.90(1.67,2.16)$ & $1.92(1.54,2.39)$ \\
Elevated diabetes risk & 4,220 & $97(2.30)$ & 37,646 & $2.58(2.11,3.14)$ & $2.60(1.99,3.39)$ \\
High diabetes risk with NGT & 2,628 & $100(3.81)$ & 23,590 & $4.24(3.48,5.16)$ & $4.28(3.29,5.57)$ \\
IFG & 913 & $108(11.83)$ & 7,464 & $14.47(11.98,17.47)$ & $14.61(11.28,18.92)$ \\
IGT & 1,306 & $286(21.90)$ & 9,936 & $28.79(25.64,32.32)$ & $29.06(23.52,35.90)$ \\
$\quad$ Isolated IGT & 765 & $133(17.39)$ & 6,151 & $21.62(18.24,25.63)$ & $21.83(17.08,27.89)$ \\
$\quad$ Combined IFG and IGT & 541 & $153(28.28)$ & 3,785 & $40.42(34.50,47.36)$ & $40.81(32.19,51.74)$ \\
One diabetic glucose value & 622 & $213(34.24)$ & 4,053 & $52.56(45.95,60.11)$ & $53.06(42.49,66.24)$ \\
\hline
\end{tabular}

A full explanation of the diabetes risk categories is given in the Methods/Study population section

Although the primary motivation and stated aim of these programmes are generally to identify people with prevalent undiagnosed diabetes, the process of screening also leads to identification of an increasing number of screen-negative (no undiagnosed diabetes present) people, who are nevertheless at various intermediate levels of increased diabetes risk. We followed a large group of people identified in a pragmatic stepwise screening programme in general practice in Denmark. Accordingly, we provide clinically relevant new evidence on the relative risk of diabetes for the graded risk groups identified by screening. As shown previously, 50\% responded to the invitation, and of those who had risk scores $\geq 5,77 \%$ attended the stepwise screening programme [20]. By

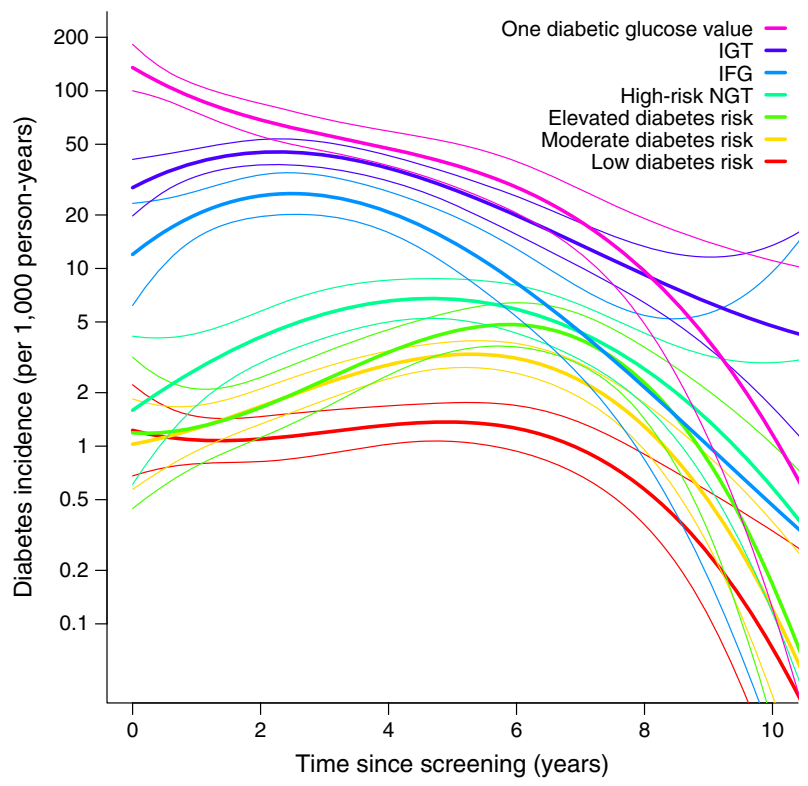

Fig. 2 Diabetes incidence rates during 10 years of follow-up for seven risk groups of the ADDITION-Denmark study (thick lines). Thin lines represent $95 \%$ CIs. A full explanation of the diabetes risk categories is given in the Methods/Study population section comparison, 53\% responded in the population-based Danish Inter99 study, which screened for diabetes and cardiovascular disease [23]. The Danish National Diabetes Register represents diagnostic practice in the real world. It is the best available diabetes register in Denmark, covering both primary care and hospitals, and it is nationwide, i.e. it covers all those with a Central Person Register number.

The incidence of diabetes using registers will inevitably be different from that defined by glucose measures or $\mathrm{HbA}_{1 \mathrm{c}}$. Hence, the diabetes incidence in the present study was lower than previously estimated by glucose measures in the ADDITION-Denmark study [16]; similar tendencies appear when comparing estimates from studies with repeated OGTT measures with estimates from register-based studies [6-13]. In general, diabetes registers are constructed according to in- and outpatient hospital diagnostic coding, reimbursement data, medical prescriptions and, to some extent, laboratory data. When comparing the Danish National Diabetes Register with a local Danish county diabetes register, both registers found a prevalence of diabetes of $4 \%$, but only $63 \%$ were the same individuals [24]. The Danish National Diabetes Register is based on accessible national registers, which do not include laboratory results, as laboratory reporting systems vary between regions in Denmark. The local register used performance of $\mathrm{HbA}_{1 \mathrm{c}}$ measurement and values above $6.6 \%(47 \mathrm{mmol} / \mathrm{mol})$ instead of ascertainment of blood glucose measures and chiropody for the diagnosis of diabetes.

The two main inclusion criteria in the Danish National Diabetes Register are ascertainment of five blood glucose values in 1 year and prescription of glucose-lowering drugs, closely followed by hospital diagnosis of diabetes in the National Patient Register [24, 25]. We excluded the two criteria on blood glucose measurements because the ADDITION-Denmark population was more likely than nonstudy populations to have frequent blood glucose 
measurements without having diabetes. This decision yielded a highly specific, but not very sensitive, diabetes definition in our study, leading to conservative estimates of diabetes incidence. Register-based studies, by definition, identify only diagnosed cases and can therefore not be expected to identify the true biological onset of diabetes. Furthermore, registers are liable to a time lag in inclusion between the date of physician diagnosis through to prescription and redemption of glucoselowering drugs. This time lag is an inherent feature of the way the diagnosis is defined in the Danish National Diabetes Register and is likely to lead to a lower than expected incidence of diabetes. These delays may be pronounced, especially for newly diagnosed people. According to the Danish Adult Diabetes Database, primarily based on data from Danish hospitals, we know that there is an under-reporting of about $15 \%$ when $\mathrm{HbA}_{1 \mathrm{c}}$ is $6.5-7.0 \%(48-53 \mathrm{mmol} / \mathrm{mol})$ and $14 \%$ for $\mathrm{HbA}_{1 \mathrm{c}}$ between $7.0 \%$ and $7.5 \%(53-58 \mathrm{mmol} / \mathrm{mol})$ in the National Danish Diabetes Register (M. E. Jørgensen, Steno Diabetes Center, Gentofte, Denmark, personal communication). Besides, poor adherence to medication is common in patients with type 2 diabetes, and a large proportion never initiate glucose-lowering treatment. These patients are not captured in the register [26], but we have no reasons to believe that there may be a systematic bias according to baseline diabetes risk in the register. Hence, although the absolute incidence rates found in our study may underestimate the true incidence, the incidence rate ratios should be representative of the population in screened general practice.

We found increasing diabetes incidence in people with IFG or IGT, which peaked after 2 years. This might to some extent reflect the follow-up of people with IFG or IGT after 1-3 years in accordance with national guidelines at the time [27, 28], and this again might also have skewed the diabetes incidence due to diagnostic bias compared with lower risk groups. It is a limitation to the interpretation of the time-dependent incidence rates that it is unknown to us how the other risk groups were followed up and what happened after the third year. However, we believe that the relative risks in our study reflect diagnostic practice in everyday life. Diabetes incidence in the intermediate-risk groups increased over time and peaked after 5-6 years. The fact that diabetes incidence in the intermediaterisk groups was not constant indicates that those screened have been followed up, even though there were no guideline recommendations at that time for individuals without IFG or IGT. Regarding the lowest risk group, diabetes incidence was low and more or less constant during the follow-up period. This is an essential finding for future healthcare planning, indicating that people with low risk scores based on risk factors for diabetes and without glucose measures need no systematic re-screening.

The risk group with one diabetic glucose value must be close to the diabetic threshold in the natural history of developing diabetes. The diabetes incidence rate in this risk group was highest shortly after the time of screening and had no later peak. Generally, the incidence rates seem to decrease markedly and broader CIs are observed in the last time period after screening, being most pronounced from 7 to 10 years. This may be explained by depletion of susceptible people over time in a closed cohort, as the most susceptible will develop diabetes soon after screening, leaving the cohort with a larger proportion of less susceptible people.

Today, the diagnosis of diabetes can be based on both blood glucose and $\mathrm{HbA}_{1 \mathrm{c}}$ [29]. In the present study, the diagnosis of diabetes at screening and during follow-up was based on the WHO 1999 criteria, before the diagnostic use of $\mathrm{HbA}_{1 \mathrm{c}}$ was implemented in Denmark in 2012. How diagnostic practice in general practice might have changed since 2012 is unknown.

In our study the stratification into risk groups was based on the design of the screening algorithm. At present, a screening algorithm in Denmark would most probably be different, by using $\mathrm{HbA}_{1 \mathrm{c}}$ rather than $\mathrm{FBG}$ or $2 \mathrm{hBG}$ and giving the following groups: low risk (low risk score), moderate risk $\left(\mathrm{HbA}_{1 \mathrm{c}}\right.$ $<6.0 \%$ [42 $\mathrm{mmol} / \mathrm{mol}])$, high risk $\left(\mathrm{HbA}_{1 \mathrm{c}} 6.0-6.4 \%\right.$ [42$47 \mathrm{mmol} / \mathrm{mol}])$, and one diabetic value $\left(\mathrm{HbA}_{1 \mathrm{c}} \geq 6.5 \%\right.$ [48 mmol $/ \mathrm{mol}]$ ). A meta-analysis of four published studies indicated that people with $\mathrm{HbA}_{1 \mathrm{c}}$ in the range 6.0-6.4\% (42-47 mmol $/ \mathrm{mol}$ ) had an incidence rate of $\mathrm{HbA}_{1 \mathrm{c}}$-defined diabetes of 25.6 per 1,000 person-years [14]. Our results show that even below the cut-off levels for IFG and IGT, the presence of diabetes risk factors is related to a higher incidence of diabetes, indicating that our understanding and implementation of diabetes prevention strategies should move beyond the glucose-centric view and consider intermediate dysglycaemia as part of a concert of risk factors.

Our documentation of relative increased future diabetes risk, especially in people with impaired glucose regulation compared with lower stratification groups, stresses the need for regular follow-up (annually) and systematic lifestyle counselling in these risk groups, as real-life preventive programmes are effective, though effectiveness varies between programmes [30]. In people with elevated risk factors for diabetes but normal glucose regulation at the time of screening, the increased risk of future diabetes underlines a need for regular re-screening for diabetes. Based on our observed time-dependent incidence rates for diabetes we suggest 3 year intervals for re-screening of these intermediate-risk groups. However, as our study is limited by its registry linkage with risk of bias in that the different risk groups may have been seen differently for follow-up by their general practitioners, further studies are needed where all risk groups in a screening programme attend the same follow-up schedule. Our study furthermore asks for evidence of possible intervention strategies, i.e. lifestyle counselling, to prevent development of diabetes in individuals with high diabetes risk but normal or intermediate glucose regulation. As high-risk 
screening or systematic case finding is already commonly recommended, these individuals are being identified and guidance on how to advise them is needed.

Conclusions The risk of future diabetes in people who screen negatively for diabetes depends on the level of risk stratification at screening: the higher the risk stratification at screening, the higher the risk of developing diabetes during 10 years. A very low incidence rate for people with low estimated diabetes risk based on risk factors alone indicates no need for rescreening at time-specific intervals, whereas groups with impaired glucose regulation have a significantly increased risk of short-term future diabetes. However, screen-negative individuals with normoglycaemia should not be ignored, as the diabetes incidence in this group increases during the first 5-6 years after screening. Although high-risk strategies differ and the results from this one cannot be extrapolated directly to other highrisk or case-finding strategies, the point of relatively increased risk of diabetes in graded risk groups applies generally. These results may support policy recommendations for prevention strategies following screening programmes.

Acknowledgements We thank B. Carstensen (Steno Diabetes Center, Gentofte, Denmark) for his advice on the statistical analyses, and all general practitioners and their staff for making this study possible.

Funding This study received funding from: the Danish Center for Evaluation and Health Technology Assessment; the Danish Research Foundation for General Practice; the counties of Aarhus, Copenhagen, Ringkoebing, Ribe, and South Jutland; the National Board of Health; the Danish Medical Research Council (no. 22-04-0390); the Danish Diabetes Association; the Danish Diabetes Academy; the A. P. Møller Foundation; Novo Nordisk A/S and Novo Nordisk Scandinavia AB; AstraZeneca; Pfizer; Servier; GlaxoSmithKline; and HemoCue. NBJ and DRW are supported by the Danish Diabetes Academy, which is funded by the Novo Nordisk Foundation.

Duality of interest SSR is principal investigator of a trial conducted by Boehringer-Ingelheim. MEJ is principal investigator of an investigatorinitiated trial sponsored by AstraZeneca. NBJ and MEJ are employed by Steno Diabetes Center A/S, a research hospital working in the Danish National Health Service and owned by Novo Nordisk A/S. Steno Diabetes Center receives part of its core funding from unrestricted grants from the Novo Foundation and Novo Nordisk A/S.

NBJ, TL, DRW, KB-J and MEJ own shares in Novo Nordisk A/S. SSR is a member of an advisory board for Novo Nordisk. Within the last 2 years TL has been member of an advisory board for AstraZeneca and is chief advisor for the Danish Diabetic Association.

Contribution statement NBJ and SSR posed the research question, designed and carried out the analyses, discussed and concluded the results and wrote the paper. MEJ and DRW contributed to the study design, interpretation of the results and review of the paper. TL, KB-J and AS designed, raised funding for and implemented the ADDITION-Denmark study, and contributed to the interpretation and review of the paper. All authors read and approved the final version of the paper. SSR is the guarantor of this work.

\section{References}

1. Knowler WC, Barrett-Connor E, Fowler SE et al (2002) Reduction in the incidence of type 2 diabetes with lifestyle intervention or metformin. N Engl J Med 346:393-403

2. Pan XR, Li GW, Hu YH et al (1997) Effects of diet and exercise in preventing NIDDM in people with impaired glucose tolerance. The Da Qing IGT and Diabetes Study. Diabetes Care 20:537-544

3. Chiasson JL, Josse RG, Gomis R et al (2002) Acarbose for prevention of type 2 diabetes mellitus: the STOP-NIDDM randomised trial. Lancet 359:2072-2077

4. Lindstrom J, Peltonen M, Eriksson JG et al (2013) Improved lifestyle and decreased diabetes risk over 13 years: long-term followup of the randomised Finnish Diabetes Prevention Study (DPS). Diabetologia 56:284-293

5. Costa B, Barrio F, Cabre JJ et al (2012) Delaying progression to type 2 diabetes among high-risk Spanish individuals is feasible in real-life primary healthcare settings using intensive lifestyle intervention. Diabetologia 55:1319-1328

6. Carstensen B, Kristensen JK, Ottosen P, Borch-Johnsen K, Steering Group of the National Diabetes Register (2008) The Danish National Diabetes Register: trends in incidence, prevalence and mortality. Diabetologia 51:2187-2196

7. Nichols GA, Schroeder EB, Karter AJ et al (2015) Trends in diabetes incidence among 7 million insured adults, 2006-2011: the SUPREME-DM project. Am J Epidemiol 181:32-39

8. Andersson T, Ahlbom A, Magnusson C, Carlsson S (2014) Prevalence and incidence of diabetes in Stockholm County 19902010. PLoS One 9:e104033

9. Rathmann W, Strassburger K, Heier M et al (2009) Incidence of type 2 diabetes in the elderly German population and the effect of clinical and lifestyle risk factors: KORA S4/F4 cohort study. Diabet Med 26:1212-1219

10. Bonora E, Kiechl S, Willeit J et al (2004) Population-based incidence rates and risk factors for type 2 diabetes in white individuals: the Bruneck study. Diabetes 53:1782-1789

11. Valdes S, Botas P, Delgado E, Alvarez F, Cadorniga FD (2007) Population-based incidence of type 2 diabetes in northern Spain: the Asturias Study. Diabetes Care 30:2258-2263

12. Soriguer F, Rojo-Martinez G, Almaraz MC et al (2008) Incidence of type 2 diabetes in southern Spain (Pizarra Study). Eur J Clin Invest 38:126-133

13. Forouhi NG, Luan J, Hennings S, Wareham NJ (2007) Incidence of type 2 diabetes in England and its association with baseline impaired fasting glucose: the Ely study 1990-2000. Diabet Med 24:200-207

14. Morris DH, Khunti K, Achana F et al (2013) Progression rates from $\mathrm{HbA1c} 6.0-6.4 \%$ and other prediabetes definitions to type 2 diabetes: a meta-analysis. Diabetologia 56:1489-1493

15. Gerstein HC, Santaguida P, Raina P et al (2007) Annual incidence and relative risk of diabetes in people with various categories of dysglycemia: a systematic overview and meta-analysis of prospective studies. Diabetes Res Clin Pract 78:305-312

16. Rasmussen S, Glümer C, Sandbaek A, Lauritzen T, Borch-Johnsen K (2008) Determinants of progression from impaired fasting glucose and impaired glucose tolerance to diabetes in a high-risk screened population: 3 year follow-up in the ADDITION study, Denmark. Diabetologia 51:249-257

17. Lauritzen T, Griffin S, Borch-Johnsen K, Wareham NJ, Wolffenbuttel BHR, Rutten G (2000) The ADDITION study: proposed trial of the cost-effectiveness of an intensive multifactorial intervention on morbidity and mortality among people with type 2 diabetes detected by screening. Int $\mathrm{J}$ Obes (Lond) 24(Suppl 3):S6-S11 
18. Glümer C, Carstensen B, Sandbaek A et al (2004) A Danish diabetes risk score for targeted screening: the Inter99 study. Diabetes Care 27:727-733

19. World Health Organization (1999) Definition, diagnosis and classification of diabetes mellitus and its complications. Report of a WHO consultation. Part 1: diagnosis and classification of diabetes mellitus. World Health Organization, Geneva

20. Christensen JO, Sandbaek A, Lauritzen T, Borch-Johnsen K (2004) Population-based stepwise screening for unrecognised type 2 diabetes is ineffective in general practice despite reliable algorithms. Diabetologia 47:1566-1573

21. Carstensen B, Kristensen JK, Marcussen MM, Borch-Johnsen K (2011) The National Diabetes Register. Scand J Public Health 39:58-61

22. Green A, Sortso C, Jensen PB, Emneus M (2015) Validation of the Danish National Diabetes Register. Clin Epidemiol 7:5-15

23. Glümer C, Jørgensen T, Borch-Johnsen K (2003) Prevalences of diabetes and impaired glucose regulation in a Danish population. The Inter99 study. Diabetes Care 26:2335-2340

24. Nielsen AA, Christensen H, Lund ED, Christensen C, Brandslund I, Green A (2014) Diabetes mortality differs between registers due to various disease definitions. Dan Med J 61:A4840
25. Danish Health and Medicines Authority (2007) The National Danish Diabetes Register. Available from sundhedsstyrelsen.dk/ publ/tidsskrifter/nyetal/pdf/2009/01_09.pdf. Accessed 5 January 2015

26. Jensen ML, Jorgensen ME, Hansen EH, Aagaard L, Carstensen B (2014) A multistate model and an algorithm for measuring longterm adherence to medication: a case of diabetes mellitus type 2 . Value Health 17:266-274

27. Royal College of General Practitioners in Denmark. Type 2 diabetes in general practice - an evidence based guideline. Royal College of General Practioners, Copenhagen, Denmark

28. Royal College of General Practitioners in Denmark. Prevention of ischemic cardiovascular disease in general practice. Royal College of General Practioners, Copenhagen, Denmark

29. American Diabetes Association (2015) (2) Classification and diagnosis of diabetes. Diabetes care 38 Suppl: S8-S16

30. Dunkley AJ, Bodicoat DH, Greaves CJ et al (2014) Diabetes prevention in the real world: effectiveness of pragmatic lifestyle interventions for the prevention of type 2 diabetes and of the impact of adherence to guideline recommendations: a systematic review and meta-analysis. Diabetes Care 37:922933 